\title{
Erratum to: Bowel sonography in sepsis with pathological correlation: an experimental study
}

\author{
Hwa-Young Kim • In-One Kim • Woo Sun Kim • \\ Gyeong Hoon Kang
}

Published online: 5 October 2010

(C) Springer-Verlag 2010

\section{Erratum to: Pediatr Radiol}

\section{DOI 10.1007/s00247-010-1806-4}

The first author's initials are incorrect in the materials and methods section, under US. The first sentence of that section should read as follows:

“A pediatric radiologist (H-YK) with 8 years' experience in pediatric radiology and 5 years' experience in bowel sonography performed transabdominal sonography with a 10-MHz linear probe (IU 22; Philips, Bothel, WA, USA)."

The online version of the original article can be found at http://dx.doi. org/10.1007/s00247-010-1806-4.

H.-Y. Kim • I.-O. Kim $(\bowtie) \cdot$ W. S. Kim

Department of Radiology, Seoul National University

College of Medicine and Institute of Radiation Medicine,

101 Daehangno, Jongno-Gu,

Seoul 110-744, South Korea

e-mail:kimio@snu.ac.kr

H.-Y. Kim

Department of Radiology, Cincinnati Children's Hospital,

Cincinnati, OH, USA

G. H. Kang

Department of Pathology, Seoul National University

College of Medicine,

Seoul, South Korea 